\title{
Is there a need to reconsider the use of metformin in COVID-19 patients with type 2 diabetes mellitus?
}

\author{
Gilbert Lazarus $^{1}$ (D) $\cdot$ Indira P. Suhardi ${ }^{1}$ Elvan Wiyarta ${ }^{1} \cdot$ Rufiah A. Rasyidah $^{1} \cdot$ Julie D. Barliana ${ }^{2}$
}

Received: 2 December 2020 / Accepted: 11 January 2021 / Published online: 2 March 2021

(C) Research Society for Study of Diabetes in India 2021

\begin{abstract}
Introduction Diabetes has been linked with poorer outcomes in coronavirus disease (COVID-19) patients. However, the question to whether continue or withdraw metformin therapy in COVID-19 patients with type 2 diabetes mellitus remains contentious. This study aims to investigate the association between metformin and poor COVID-19 outcomes.

Methods Eligible studies published up to 21 October 2020 were included and appraised for validity, importance, and applicability. The included studies were further ranked according to the level of evidence (LOE).

Results Nine studies were included for further assessments, of which seven studies stated that metformin was not associated with poor COVID-19 outcomes (LOE II-V), while the other two with poorer designs stated otherwise (LOE V). Although metformin may increase the risk of developing acidosis and lactic acidosis (LOE IV), the observed risks were more accentuated in patients with severe COVID-19 disease or kidney impairment and in patients with $>2$ daily metformin doses. Interestingly, one study revealed that metformin may even yield therapeutic role in reducing the risk of COVID-19 mortality (LOE II), although further studies are required to confirm these findings.

Conclusions Our findings indicated that metformin may be safely continued in COVID-19 patients. The benefit of metformin therapy with simultaneous continuous monitoring of COVID-19 severity and kidney function may outweigh the risks of lactic acidosis, of which incidence is relatively rare.
\end{abstract}

Keywords COVID-19 $\cdot$ Metformin $\cdot$ Type 2 diabetes mellitus $\cdot$ Prognosis

\section{Clinical scenario}

A 45 -years-old man with a 5-year history of type 2 diabetes mellitus (T2DM) came to the hospital with mild-to-moderate coronavirus disease 2019 (COVID-19) infection. His T2DM had been well-controlled by metformin $500 \mathrm{mg}$ bid, and his laboratory panel revealed a $\mathrm{HbA}_{1 \mathrm{C}}$ level of $43 \mathrm{mmol} / \mathrm{mol}$ and a random blood glucose level of $8.6 \mathrm{mmol} / \mathrm{L}$, while the other tests were unremarkable.

Recent consensuses have recommended diabetic patients to temporarily cease metformin treatment during the course of

Gilbert Lazarus

gilbert.lazarus@ui.ac.id

1 Faculty of Medicine, Universitas Indonesia, Jl. Salemba Raya No. 6, RW 5, Kenari, Kec. Senen, Kota Jakarta Pusat, Jakarta 10430, Indonesia

2 Department of Ophthalmology, Faculty of Medicine Universitas Indonesia, Jakarta, Indonesia
COVID-19 disease [1, 2]. However, the patient expressed his concerns that he wishes to stay with the current medication. Acknowledging the patient's wishes, the general practitioner wonders whether metformin had any effect on COVID-19 outcomes.

\section{Introduction}

The disease burden of the COVID-19 pandemic has perpetually surged with millions of cases and deaths. Diabetes has been strongly associated with poor prognosis in COVID-19 patients. [3] However, the management strategy of diabetic COVID-19 patients remains contentious as previous reports showed conflicting results $[1,4]$. This raises concerns about the safety of metformin, the gold standard first-line antidiabetic treatment [5], in treating COVID-19-infected T2DM patients. Hence, we studied the evidence linking metformin and poor COVID-19 outcomes in T2DM patients, thus 
providing guidance for clinicians to make real-time treatment decisions in such cases.

\section{Clinical question}

Is metformin therapy associated with poor prognosis in COVID-19-infected T2DM patients?

\section{Methods}

\section{Search strategy and eligibility criteria}

We comprehensively searched PubMed, Embase, CENTRAL, EBSCO MEDLINE, CINAHL, and gray literature databases (Google Scholar, ProQuest, Scopus, preprints) for studies investigating the effect of metformin on COVID19 outcomes, including mortality, severity, and other prognostic surrogates, published up to 21 October 2020 using keywords listed on Supplementary Table S1. Records were screened against the eligibility criteria listed on Fig. 1, and no language restrictions were applied.

\section{Critical appraisal}

The included studies were further appraised for validity, importance, and applicability using a standardized tool [8]. Critical appraisal and literature searches were conducted by two pairs of independent adjudicators (GL and RAR, EW and IPS), with discordant judgments resolved by consensus between the authors. Lastly, the studies were ranked according to their level of evidence (LOE) [9].

\section{Results}

The initial search yielded 1094 records, of which 16 were retrieved following thorough reviews. Among them, two systematic reviews (SRs) were excluded as one had overlapping studies with smaller sample size [10], and another one pooled the unadjusted rather than the adjusted estimates [6]. In addition, five observational studies were also excluded as they were already included in the SR. Therefore, nine studies comprising of one SR of cohort studies, six retrospective cohort studies, one case series, and one case-control study qualified
Fig. 1 Diagram flow illustrating the literature search and selection. ${ }^{a}$ Although one of the studies included in a systematic review by Hariyanto et al. [6] was not included in the systematic review by Kow et al. [7], the study only pooled unadjusted rather than adjusted estimates; thus we decided to exclude the study by Hariyanto et al. [6] and assessed the non-duplicate study separately. CENTRAL, Cochrane Central Register of Controlled Trials; CINAHL, Cumulative Index to Nursing and Allied Health Literature. COVID-19, coronavirus disease 2019; SSRN, Social Science Research Network; T2DM, type 2 diabetes mellitus; WHO, World Health Organization COVID-19

Research Database

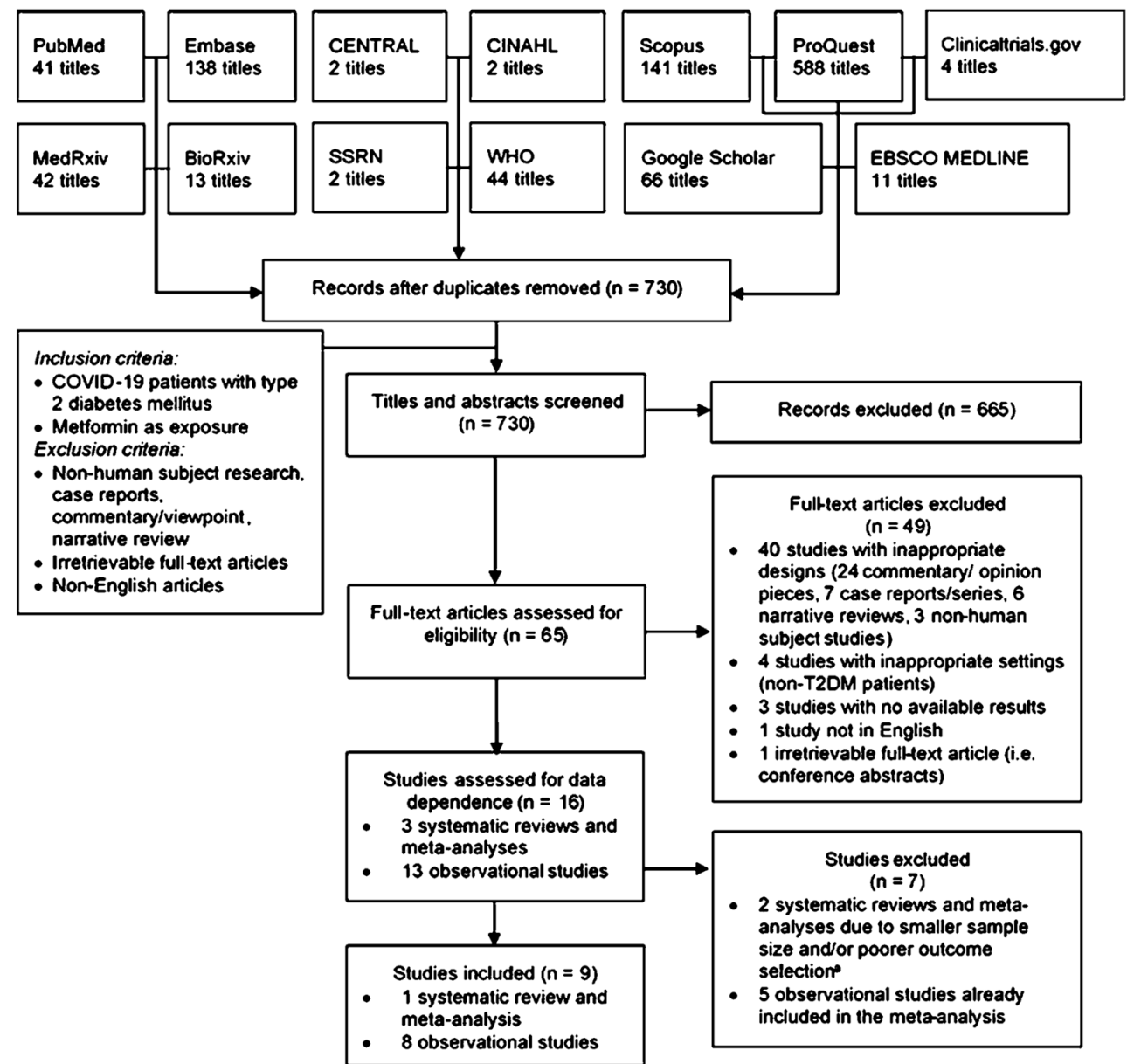




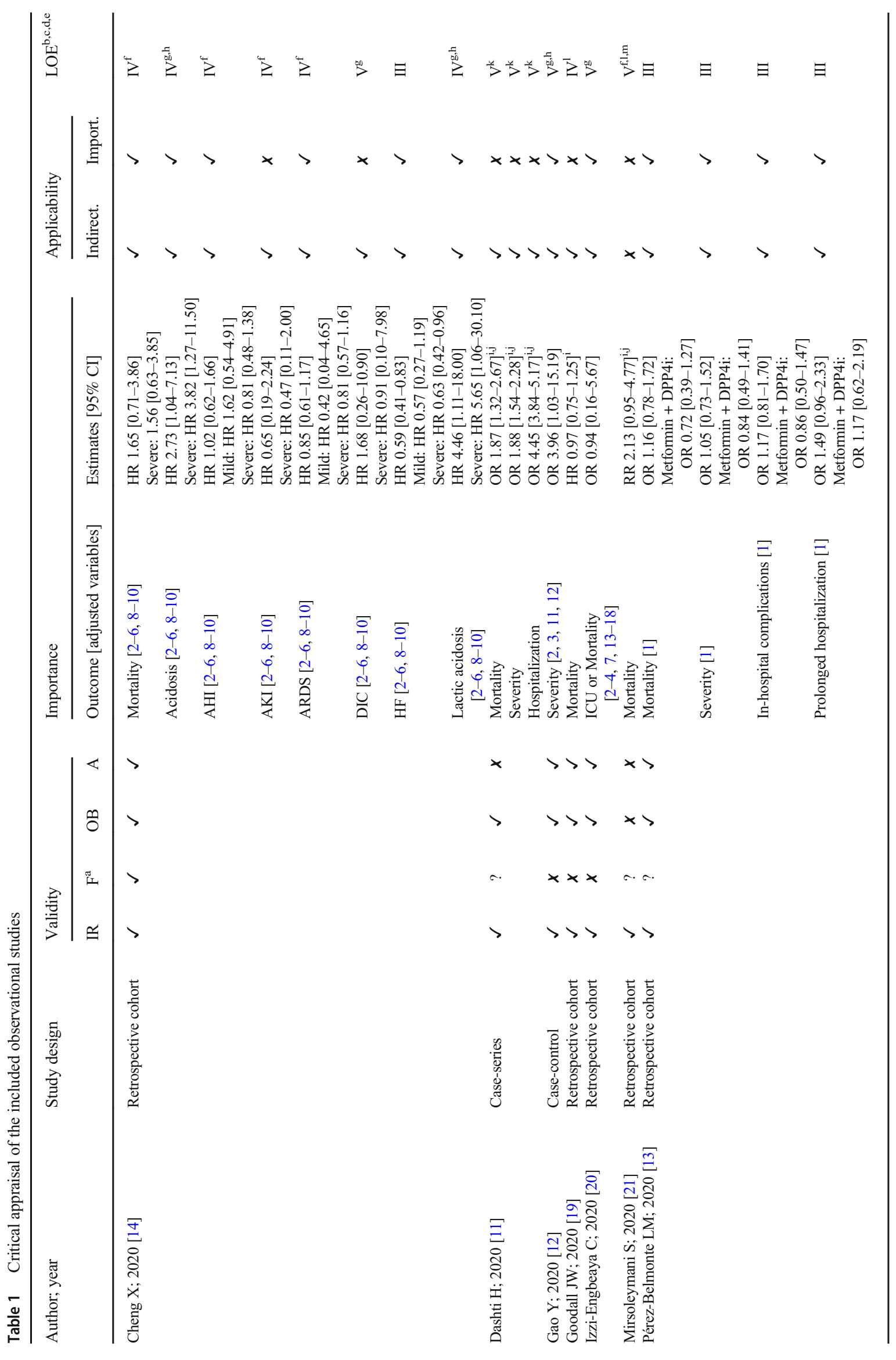




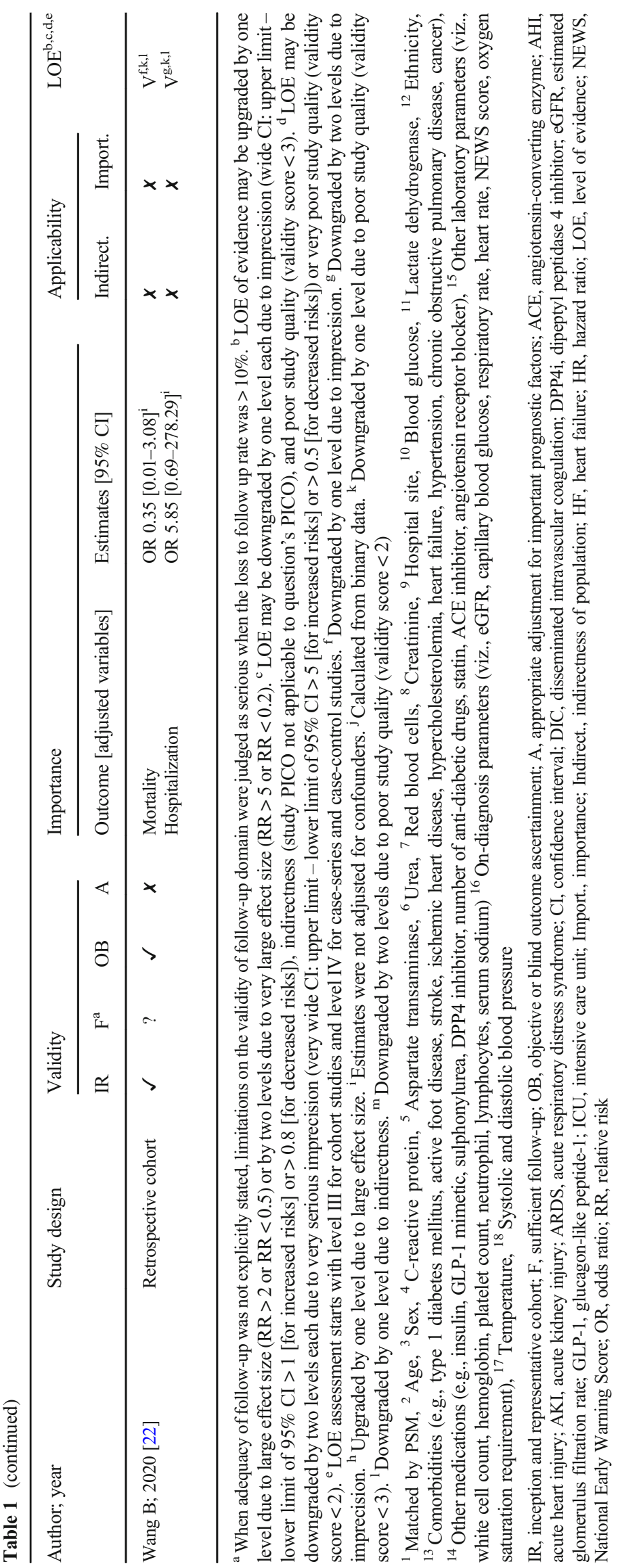


Table 2 Critical appraisal of the included systematic review

\begin{tabular}{|c|c|c|c|c|c|c|c|c|c|c|}
\hline \multirow[t]{2}{*}{ Author; year } & \multirow[t]{2}{*}{ Study design } & \multicolumn{5}{|c|}{ Validity } & \multicolumn{3}{|c|}{ Importance } & \multirow[t]{2}{*}{$\mathrm{LOE}^{\mathrm{a}, \mathrm{b}, \mathrm{c}}$} \\
\hline & & PICO & $\mathrm{F}$ & $\mathrm{A} 1$ & $\mathrm{~A} 2$ & $\mathrm{~T}$ & Outcome & Estimates $[95 \% \mathrm{CI}]$ & Heterogeneity & \\
\hline Kow CS; 2020 [7] & SR of cohort studies & $\checkmark$ & $x$ & $\checkmark$ & $\checkmark$ & $\checkmark$ & Mortality & OR $0.62[0.43-0.89]$ & $I^{2}=29 \% ; p=0.23$ & $\mathrm{II}^{\mathrm{d}, \mathrm{e}}$ \\
\hline
\end{tabular}

${ }^{a} \mathrm{LOE}$ of evidence may be upgraded by one level due to large effect size $(\mathrm{RR}>2$ or $\mathrm{RR}<0.5)$ or by two level due to very large effect size $(\mathrm{RR}>5$ or $\mathrm{RR}$ <0.2). ${ }^{\mathrm{b}}$ LOE may be downgraded by one level each due to imprecision (wide CI: upper limit - lower limit of $95 \%$ CI $>1$ [for increased risks] or $>0.8$ [for decreased risks]), indirectness (study PICO not applicable to question's PICO), inconsistency $\left(I^{2}>50 \%\right.$ or $p<0.10$ ), and poor study quality (validity score <4). ${ }^{\mathrm{c}} \mathrm{LOE}$ may be downgraded by two levels each due to very serious imprecision (very wide CI: upper limit - lower limit of $95 \% \mathrm{CI}>5$ [for increased risks] or $>0.5$ [for decreased risks]), very serious inconsistency $\left(I^{2}>75 \%\right.$ or $p<0.01$ ), or very poor study quality (validity score $\left.<2\right)$. ${ }^{\mathrm{d}} \mathrm{LOE}$ assessment starts with level III. ${ }^{\mathrm{e}}$ Upgraded by one level due to systematic review design

$\checkmark$, no serious limitation; $\boldsymbol{x}$, serious limitation; PICO, clear statement of review question; F, comprehensive strategy to find eligible studies; A1, appropriate eligibility criteria; A2, appropriate risk of bias or methodological quality assessment; $\mathrm{T}$, appropriate strategy to total up the summary of findings; CI, confidence interval; I ${ }^{2}$, I-squared value; LOE, level of evidence; OR, odds ratio; SR, systematic review

for inclusion. Details on the literature search process are illustrated by Fig. 1.

We discovered that most studies stated that metformin use was not associated with poor COVID-19 outcomes, whereas only two studies stated otherwise $[11,12]$. However, both studies were underpowered to determine causalities due to poorer designs. Furthermore, one study [11] did not adjust for important confounders, and another one [12] yielded excessively wide confidence intervals (CIs), thus further diminishing the LOE (LOE V; Table 1). Among the included studies, four were deemed not clinically important as the results were not adjusted for confounders, while another one was judged inapplicable as they only included COVID-19 patients with multiple myeloma.

Interestingly, Kow et al. discovered that metformin use was associated with a lower rate of COVID-19 mortality (odds ratio [OR] 0.62, 95\% CI 0.43-0.89, LOE II; Table 2) [7]. Although some study-specific estimates were inconsistent, the model only yielded non-significant low heterogeneity $\left(I^{2}=29 \%, p=0.23\right)$ [7], hence resulting in the judgment of LOE II. This was further corroborated by Pérez-Belmonte et al. who stated that either metformin monotherapy or its combination with dipeptyl peptidase-4 inhibitor remained safe for diabetic COVID-19 patients (LOE III) [13] - further ascertaining the findings. On the other hand, Cheng et al. stated that metformin was associated with increased risk of acidosis and lactic acidosis (LOE IV), although the effect was more accentuated in patients with severe COVID-19 infection (oxygen saturation $<93 \%$ or neutrophil-to-lymphocyte ratio $>$ 3.13) or kidney impairment (estimated glomerular filtration rate $<60 \mathrm{~mL} / \mathrm{min} / 1.73 \mathrm{~m}^{2}$ ), and in patients taking $>2$ daily metformin doses [14] (Table 1).

\section{Discussion}

The question to whether continue or adjourn metformin therapy in COVID-19 patients remained relevant during the pandemic. Recent consensus has recommended postponing metformin therapy due to the risk of dehydration and lactic acidosis $[1,2]$; however, some others argued that the impending benefits of metformin may outweigh the risks of developing adverse events as their incidence is relatively rare $[15,16]$. According to our findings, it is plausible to continue metformin therapy while simultaneously increasing vigilance on the patient's COVID-19 severity and kidney function. Furthermore, our patient was not at higher risks of developing acidosis as his COVID-19 disease was not severe and his renal panel was unremarkable. In addition, his daily metformin doses were still within the safe limit [14], thus further inclining towards the use of metformin. Furthermore, it is also important to heed to patients' preference as it may substantially affect treatment adherence and outcome. [17] This is saliently important considering that poorer glycemic control has been associated with poor COVID-19 prognosis [18], implying that the benefits of continuing metformin may also outweigh the risk of noncompliance. Despite the favorable findings, further prospective studies are required to establish a firm evidence as the included studies yielded predominantly low-to-moderate LOE.

Further extrapolating our findings, recent reports have suggested the potential therapeutic role of metformin in COVID$19[7,10]$. Several mechanisms have been hypothesized, including its ability to induce phosphorylation of angiotensinconverting enzyme-2 receptors to inhibit viral entry and replication [10]. Furthermore, metformin yields potent antioxidative, anti-inflammatory, and pulmoprotective properties, thus further suggesting the impending benefits [10]. Nonetheless, further studies are required to substantiate these premises.

\section{Conclusion}

In summary, our findings indicated that metformin may be safely continued in diabetic COVID-19 patients. However, several factors need to be considered when assessing the 
benefits and risks of continuing metformin therapy, including COVID-19 severity, kidney function, and glycemic control. Rather than indifferently deferring metformin therapy, it is more imperative to continuously monitor the patient's COVID-19 disease course and renal function, deterioration of which may predict poor COVID-19 outcomes.

Supplementary Information The online version contains supplementary material available at https://doi.org/10.1007/s13410-021-00924-w.

Authors' contribution All authors contributed to idea conception, data abstraction, and manuscript draft. GL developed the methodology and visualized the data. GL and JDB reviewed and edited the initial manuscript draft. All authors have read and approved the final manuscript for submission.

Funding This research did not receive any specific grant from funding agencies in the public, commercial, or not-for-profit sectors.

Data availability The data that supports the findings of this study are available in the supplementary material of this article.

\section{Declarations}

Conflict of interest The authors declare no conflict of interest.

\section{References}

1. Bornstein SR, Rubino F, Khunti K, Mingrone G, Hopkins D, Birkenfeld AL, et al. Practical recommendations for the management of diabetes in patients with COVID-19. Lancet Diabetes Endocrinol Elsevier Ltd. 2020;8:546-50.

2. Hartmann-Boyce J, Morris E, Goyder C, Kinton J, Perring J, Nunan D, et al. Diabetes and COVID-19: risks, management, and learnings from other national disasters. Diabetes Care. 2020;43:1695-703.

3. World Health Organization. Clinical management of COVID-19 interim guidance 27 May 2020. Geneva: World Health Organization; 2020.

4. Hussain A, Bhowmik B, do Vale Moreira NC. COVID-19 and diabetes: Knowledge in progress. Diabetes Res Clin Pract. Elsevier Ireland Ltd; 2020;108142.

5. Gnesin F, Thuesen ACB, Kähler LKA, Madsbad S, Hemmingsen B. Metformin monotherapy for adults with type 2 diabetes mellitus. Cochrane Database Syst Rev. John Wiley and Sons Ltd; 2020;2020.

6. Hariyanto TI, Kurniawan A. Metformin use is associated with reduced mortality rate from coronavirus disease 2019 (COVID-19) infection. Obes Med. 2020;19:100290.

7. Kow CS, Hasan SS. Mortality risk with preadmission metformin use in patients with COVID-19 and diabetes: a meta-analysis. J Med Virol. 2020:1-3.

8. Critical appraisal of prognostic studies. Oxford: Centre for Evidence-Based Medicine, University of Oxford; 2020 [cited
2020 Oct 28]. Available from: https://www.cebm.ox.ac.uk/ resources/ebm-tools/critical-appraisal-tools

9. OCEBM Levels of Evidence Working Group. The Oxford levels of evidence 2. Oxford: Oxford Centre for Evidence-Based Medicine; 2011 [cited 2020 Oct 27]. Available from: https://www.cebm.ox. ac.uk/resources/levels-of-evidence/ocebm-levels-of-evidence

10. Scheen AJ. Metformin and COVID-19: From cellular mechanisms to reduced mortality. Diabetes Metab. 2020/08/05. 2020.

11. Dashti HT, Bates DW, Roche EC, Fiskio JM, Mora S, Demler OV, et al. Clinical characteristics and severity of COVID-19 disease in patients from Boston area hospitals. medRxiv. 2020. https://doi.org/ 10.1101/2020.07.27.20163071.

12. Gao Y, Liu T, Zhong W, Liu R, Zhou H, Huang W, et al. Risk of metformin in patients with type 2 diabetes with COVID-19: a preliminary retrospective report. Clin Transl Sci. 2020;13(6):1055-9.

13. Pérez-Belmonte LM, Torres-Peña JD, López-Carmona MD, AyalaGutiérrez M, Fuentes-Jiménez F, Huerta LJ, et al. Impact of glucose-lowering drugs on mortality and other adverse outcomes in patients with type 2 diabetes admitted for COVID-19. SSRN Electron J. 2020. https://doi.org/10.2139/ssrn.3666251.

14. Cheng X, Liu YM, Li H, Zhang X, Lei F, Qin JJ, et al. Metformin is associated with higher incidence of acidosis, but not mortality, in individuals with COVID-19 and pre-existing type 2 diabetes. Cell Metab. United States: Cell Press. 2020;32:537-547.e3.

15. Chen X, Guo H, Qiu L, Zhang C, Deng Q, Leng Q. Immunomodulatory and antiviral activity of metformin and its potential implications in treating coronavirus disease 2019 and lung injury. Front Immunol. Frontiers Media S.A.; 2020.

16. Malhotra A, Hepokoski M, McCowen KC, Y-J Shyy J. ACE2, Metformin, and COVID-19. iScience. Elsevier Inc. 2020;101425: 23.

17. Brown MT, Bussell JK. Medication adherence: WHO cares? Mayo Clin. Proc. Elsevier Ltd. 2011;304-14.

18. Lazarus G, Audrey J, Wangsaputra VK, Tamara A, Tahapary DL. High admission blood glucose independently predicts poor prognosis in COVID-19 patients: A systematic review and dose-response meta-analysis. Diabetes Res Clin Pract. 2021;171:108561.

19. Goodall JW, Reed TAN, Ardissino M, Bassett P, Whittington AM, Cohen DL, et al. Risk factors for severe disease in patients admitted with COVID-19 to a hospital in London, England: A retrospective cohort study. medRxiv. https://doi.org/10.1101/2020.09.24. 20200337.

20. Izzi-Engbeaya C, Distaso W, Yang W, Idowu O, Kenkre JS, Shah RJ, et al. Severe COVID-19 and diabetes: A retrospective cohort study from three London teaching hospitals. medRxiv. 2020. https://doi.org/10.1101/2020.08.07.20160275.

21. Mirsoleymani S, Nekooghadam SM. Risk factors for severe Coronavirus Disease 2019 (COVID-19) among Iranian patients: Who was more vulnerable? SSRN Electron J. 2020. https://doi. org/10.2139/ssrn.3566216.

22. Wang B, Van Oekelen O, Mouhieddine TH, Del Valle DM, Richter $\mathrm{J}$, Cho HJ, et al. A tertiary center experience of multiple myeloma patients with COVID-19: Lessons learned and the path forward. J Hematol Oncol. 2020;13:94.

Publisher's note Springer Nature remains neutral with regard to jurisdictional claims in published maps and institutional affiliations. 